\title{
Analysis of Experimental Measurements of PWR Fresh and Spent Fuel Assemblies Using Self-Interrogation Neutron Resonance Densitometry
}

Adrienne M. LaFleur ${ }^{{ }^{*}}$ and Howard O. Menlove ${ }^{\mathrm{a}}$

${ }^{a}$ Nuclear Engineering and Nonproliferation Division, Los Alamos National Laboratory, P.O. Box 1663 MS E540, Los Alamos, NM 87545, USA

alafleur@lanl.gov; hmenlove@lanl.gov;

\section{Corresponding author:}

Name: Adrienne M. LaFleur

Postal Address: Los Alamos National Laboratory, P.O. Box 1663, MS E540, Los Alamos, NM 87545-0001, USA

Phone: +1-505-606-0443

Fax: $+1-505-665-4433$

Email: alafleur@lanl.gov 


\section{Analysis of Experimental Measurements of PWR Fresh and Spent Fuel Assemblies Using Self-Interrogation Neutron Resonance Densitometry}

Abstract

Self-Interrogation Neutron Resonance Densitometry (SINRD) is a new NDA technique that was developed at Los Alamos National Laboratory (LANL) to improve existing nuclear safeguards measurements for LWR fuel assemblies. The SINRD detector consists of four fission chambers (FCs) wrapped with different absorber filters to isolate different parts of the neutron energy spectrum and one ion chamber (IC) to measure the gross gamma rate. As a result, two different techniques can be utilized using the same SINRD detector unit and hardware. These techniques are the Passive Neutron Multiplication Counter (PNMC) method and the SINRD method. The focus of the work described in this paper is the analysis of experimental measurements of fresh and spent PWR fuel assemblies that were performed at LANL and the Korea Atomic Energy Research Institute (KAERI), respectively, using the SINRD detector. The purpose of these experiments was to assess the following capabilities of the SINRD detector: 1) reproducibility of measurements to quantify systematic errors, 2) sensitivity to water gap between detector and fuel assembly, 3) sensitivity and penetrability to the removal of fuel rods from the assembly, and 4) use of PNMC/SINRD ratios to quantify neutron multiplication and/or fissile content. The results from these simulations and measurements provides valuable experimental data that directly supports safeguards research and development (R\&D) efforts on the viability of passive neutron NDA techniques and detector designs for partial defect verification of spent fuel assemblies.

Keywords: non-destructive assay; spent fuel verification; nuclear safeguards; self-interrogation neutron resonance densitometry; neutron detector;

\section{Introduction}

28 The development of non-destructive assay (NDA) capabilities to improve verification of spent 29 fuel has become increasingly important with the worldwide expansion of nuclear power, 30 adoption of enhanced safeguards criteria for spent fuel verification, and recent efforts by the 31 International Atomic Energy Agency (IAEA) to incorporate an integrated safeguards regime [1]. 32 Under comprehensive safeguards agreements, states declare the nuclear material composition of 33 the LWR spent fuel based on the operating records of the reactors and results of reactor core 34 simulations. Declarations are formulated per spent fuel item. In addition, reactor operators 
provide operating records and irradiation histories for each fuel assemblies. For routine verification, the IAEA deploys verification measures meant to confirm the presence of declared items in the spent fuel storage by means of continuous $\mathrm{C} / \mathrm{S}$ measures or approved nondestructive verification supporting attribute tests. During an onsite inspection, IAEA inspectors verify the spent fuel inventory declared by the facility operator via item accounting and, when needed, use the appropriate technology to measure a sample of the assemblies to ensure the validity of the operator's accountancy system [2].

As opposed to simple attribute test which is normally sufficient, under special circumstances such as loss of continuity of knowledge during open core periods or when spent fuel is transferred to dry storage, the IAEA engages more powerful verification methods supporting the independent verification of the irradiation history or the completeness of the fuel assembly (i.e. the absence of pin removal or substitution). Avenues for improvement of current spent fuel verification methods include: the ability to verify LWR spent fuel assemblies independent of the operator's declaration and the existence of a measurement method that can be used for all types of fuel assemblies regardless of initial fuel enrichment, burnup, or cooling time [3,4].

\subsection{Objectives}

Self-Interrogation Neutron Resonance Densitometry (SINRD) is a new NDA technique that was developed at Los Alamos National Laboratory (LANL) to improve existing nuclear safeguards measurements for LWR fuel assemblies. The basic physics concept of the SINRD measurement technique was originally developed in 1968 at LANL using the name, Self-Indication Neutron Resonance Absorption Densitometry (SINRAD). Two experiments were performed in 1968 and 1969 with SINRAD which used a reactor beam as the interrogating neutron source to measure the fissile concentration in ${ }^{235} \mathrm{U}$ and ${ }^{239} \mathrm{Pu}$ metal plates and MOX fuel rods, respectively $[5,6,7]$. The results from these experiments demonstrate that SINRAD can accurately measure the fissile content in both metal plates and MOX fuel rods. The primary objective of this research was to develop the same basic physics signature as SINRAD but applied to LWR spent fuel assemblies (SFAs). However, in spent fuel, there is an adequate neutron source from the spontaneous fission of ${ }^{244} \mathrm{Cm}$ to be self-interrogating and no reactor beam is necessary. Thus, the original name was modified to the current name, Self-Interrogation Neutron Resonance Densitometry, for spent fuel 
applications to reflect the different neutron sources. It is important to note that SINRD can also be used to measure fresh FAs. Since the spontaneous fission rate of ${ }^{238} \mathrm{U}$ is very low, a ${ }^{252} \mathrm{Cf}$ source can be used to reduce the required count time. Recent interest in this approach was stimulated by an IAEA request related to spent fuel verification. The main application of SINRD is for use at a spent fuel storage facility for measurements in water, although SINRD could also be used for measurements in different mediums, such as air or sodium and at reprocessing facilities that have spent fuel pools [8].

The focus of the work described in this paper is on the experimental measurements of fresh and spent PWR fuel assemblies were performed at LANL and the Korea Atomic Energy Research Institute (KAERI), respectively, using the SINRD detector. SINRD has the potential to be a lowcost, robust hardware system deployed for verification of spent fuel assemblies. In addition, SINRD could also be easily integrated with a Fork detector or ${ }^{252} \mathrm{Cf}$ Interrogation with Prompt Neutron Detection (CIPN) detector given the complimentary nature of the hardware. The purpose of these experiments was to assess the following capabilities of the SINRD detector:

1) reproducibility of measurements to quantify systematic errors

2) sensitivity to water gap between detector and fuel assembly (FA)

83

$$
\text { 3) sensitivity and penetrability to the removal of fuel rods from the assembly }
$$

4) use of different fission chamber (FC) ratios to quantify neutron multiplication and/or fissile content in the FA

\subsection{Theory and Background}

91 The neutron resonance cross-section structure is unique for each fissile isotope such as ${ }^{235} \mathrm{U}$,

$92{ }^{239} \mathrm{Pu}$, and ${ }^{241} \mathrm{Pu}$, and the resonance structure can provide a signature for the measurement of 93 these materials of importance for safeguards and non-proliferation. The sensitivity of SINRD is 94 based on using the same fissile materials in the sample and fission chamber because the effect of 95 resonance absorption in the transmitted flux is amplified by the corresponding $(n, f)$ reaction 
96 peaks in the fission chamber. For instance, a ${ }^{235} \mathrm{U}$ fission chamber has a high sensitivity to the

97 neutron resonance absorption in ${ }^{235} \mathrm{U}$ present in the sample, and similarly for other fissile

98 isotopes. SINRD uses spontaneous fission neutrons from ${ }^{244} \mathrm{Cm}$ to self-interrogate the spent fuel

99 pins. The concentration of ${ }^{235} \mathrm{U}$ and ${ }^{239} \mathrm{Pu}$ in the spent fuel is then determined by measuring the

100 distinctive resonance absorption lines from ${ }^{235} \mathrm{U}$ and ${ }^{239} \mathrm{Pu}$ using ${ }^{235} \mathrm{U}$ and/or ${ }^{239} \mathrm{Pu}$ fission

101 chambers (FCs) placed adjacent to the side of the fuel assembly. Thus, the self-interrogation

102 signature is a result of having the same fissile material in the FC and the sample [9].

103

104 In Fig. 1, the ${ }^{239} \mathrm{Pu}$ fission cross-section is compared to the resonance absorption lines in the 105 neutron flux after transmission through a $0.11-\mathrm{mm}$ Gd filter and ${ }^{239} \mathrm{Pu}$ metal samples $0.25-\mathrm{mm}$ 106 and 2.5-mm thick. It is important to note that as the sample thickness increases, the self107 interrogation signature decreases due to self-shielding effects from saturation of the large ${ }^{239} \mathrm{Pu}$ 108 fission resonance at $0.3-\mathrm{eV}$ [5]. The results shown for the transmitted flux through ${ }^{239} \mathrm{Pu}$ metal 109 samples of different thicknesses were obtained from MCNP simulations and the ${ }^{239} \mathrm{Pu}$ fission 110 cross-section was obtained from the JANIS ENDF-VII cross-section database [10]. 


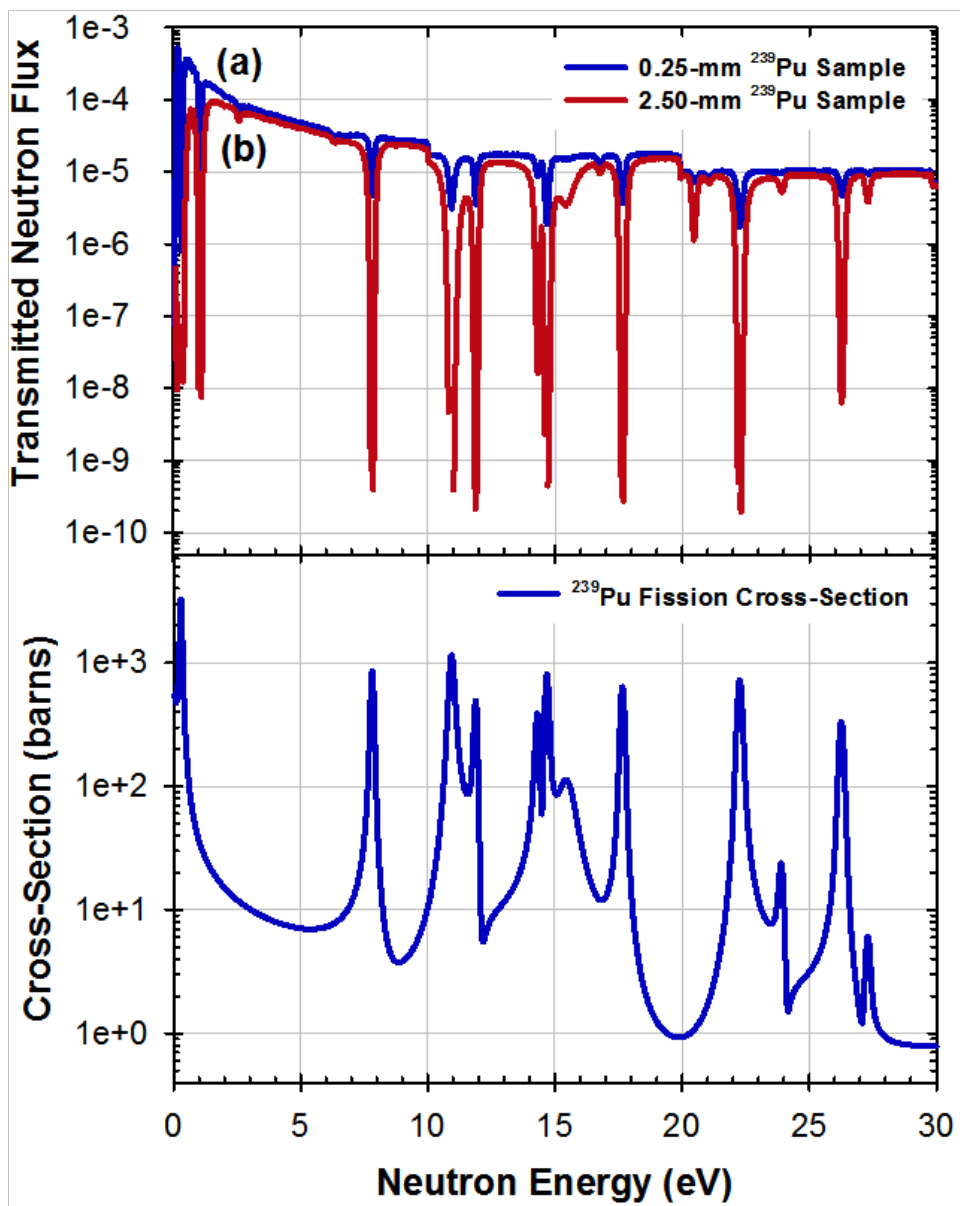

Fig. 1. Comparison of absorption lines in neutron flux after transmission through Gd filter and (a) 0.25 $\mathrm{mm}$ and (b) $2.5-\mathrm{mm}{ }^{239} \mathrm{Pu}$ metal sample (upper plot) to ${ }^{239} \mathrm{Pu}$ fission cross-section (bottom plot).

\section{Design of SINRD Detector}

118 The SINRD detector system was designed for measuring LWR fuel assemblies. Since the fuel 119 assemblies considered in this research have square lattices, we designed the SINRD detector unit 120 to be rectangular. The SINRD detector unit consists of one ion chamber (IC) and the following 121 four FCs:

- Bare ${ }^{235} \mathrm{U}$ FC: measures thermal neutrons leaking from the FA

- Boron Carbide $\left(\mathrm{B}_{4} \mathrm{C}\right){ }^{235} \mathrm{U}$ FC: measures fast neutrons leaking from the FA

- Gd covered ${ }^{235} \mathrm{U}$ FC: measures neutrons above 0.13-eV leaking from the FA

- Cd covered ${ }^{235} \mathrm{U}$ FC: measures neutrons above 1.25-eV leaking from the FA 
127 It is important to note that two different techniques can be utilized using the same SINRD 128 detector unit and hardware. These techniques are the Passive Neutron Multiplication Counter 129 (PNMC) method and the SINRD method. The PNMC method uses the ratio of the fast-neutron 130 emission rate to the thermal-neutron emission rate to quantify the neutron multiplication of the 131 item. It should also be noted that throughout the rest of this paper, we refer to the $\mathrm{B}_{4} \mathrm{C} \mathrm{FC}$ as 132 FFM (or Fast Flux Monitor) and the Bare ${ }^{235} \mathrm{U}$ FC as TFM (or Thermal Flux Monitor). The 133 neutron flux entering the detector pod is measured using two ${ }^{235} \mathrm{U}$ FCs that have differential 134 shielding to isolate fast and thermal energies:

- Thermal Flux Monitors (TFM) - Bare ${ }^{235}$ U FC located next to fuel assembly and measures the entire neutron flux spectrum with thermal neutron domination, and

- $\quad$ Fast Flux Monitors (FFM) - located behind 1-cm of $\mathrm{B}_{4} \mathrm{C}$ and measures the fast neutron flux above the $\mathrm{B}_{4} \mathrm{C}$ absorption cutoff energy (3.8-keV).

The fast-neutron emission rate is proportional to the neutron multiplication in the fuel assembly and the ${ }^{244} \mathrm{Cm}$ ingrowth for spent fuel; whereas, the thermal-neutron leakage is suppressed by the 141 fissile material absorption in the assembly.

143 The SINRD method uses the Gd and Cd covered ${ }^{235} \mathrm{U}$ FCs to measure the resonance absorption 144 from ${ }^{235} \mathrm{U}$ and ${ }^{239} \mathrm{Pu}$ in fresh and spent fuel. The thickness of each of these absorber filters was 145 chosen based on the desired absorption cutoff energy relative to the ${ }^{235} \mathrm{U}$ fission cross-section. 146 We chose to use $0.025-\mathrm{mm}$ thick Gd with a cutoff energy of $0.13-\mathrm{eV}$ and 3-mm thick Cd with a 147 cutoff energy of $1.25-\mathrm{eV}$. The transmitted flux through $\mathrm{Gd}, \mathrm{Cd}$, and $\mathrm{B}_{4} \mathrm{C}$ relative to the ${ }^{235} \mathrm{U}$ 148 fission cross-section is shown in Fig. 2. Based on the location of the Gd and Cd absorption cut149 off energies, we see that the thick Cd filter $(3.0 \mathrm{~mm})$ absorbs the majority of neutrons in the low 150 energy region of the ${ }^{235} \mathrm{U}$ and ${ }^{239} \mathrm{Pu}$ fission resonances whereas the thin $\mathrm{Gd}$ filter $(0.025 \mathrm{~mm})$ 151 transmits the majority of these lower energy neutrons. 




Fig. 2. Cut-off energies of SINRD filters relative to the ${ }^{235} \mathrm{U}$ and ${ }^{239} \mathrm{Pu}$ fission cross-sections.

155 The SINRD detector unit is located adjacent to the assembly and approximately 12.1-cm high, 156 11.8-cm long, and 24.7-cm wide. Fig. 3 shows the SINRD detector unit modeled in MCNP and 157 the actual SINRD unit that will be used in experiment.
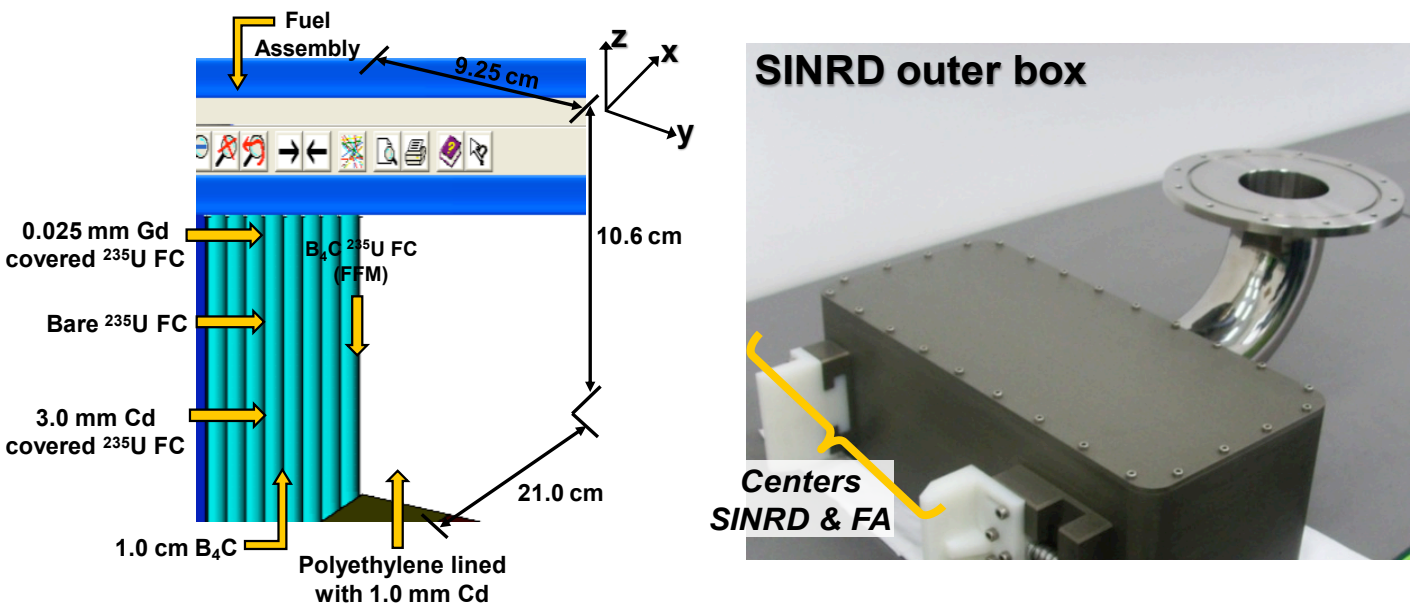

Fig. 3. SINRD detector modeled in MCNP and actual SINRD detector used in experiment.

161 To increase counting statistics, the FFM was embedded in polyethylene to thermalize the fast 162 neutrons that penetrated the boron shielding. The polyethylene was covered with $1.0-\mathrm{mm}$ of $\mathrm{Cd}$ 163 to reduce the background from reflected thermal neutrons reentering the detector from the back 
164 and sides. This also reduces the number of unknowns we are trying measure because the neutron

165 source strength and detector-fuel assembly coupling cancels in the ratio.

\section{Fresh Fuel Measurements}

168 The fresh fuel measurements were performed in water using a 161 gallon water tank and the 169 LANL reference PWR 15x15 fresh LEU fuel assembly [11]. This fuel assembly has a standard 170 PWR 15x15 grid, typical of commercial PWRs, that contains 204 fuel rods and 21 open

171 channels. The measured response from this assembly has been compared to similar fuel assembly 172 measurements at several fuel fabrication facilities and the agreement has been within $\pm 2 \%$ [12].

\subsection{Experimental Setup}

175 The empty $15 \times 15$ PWR fuel assembly rack was placed in the center of the 161 gallon tank and 176 loaded with 120 LEU (yellow) and 84 DU (red) fuel rods according to the configuration shown 177 in Fig. 4. Last the tank was filled with water.

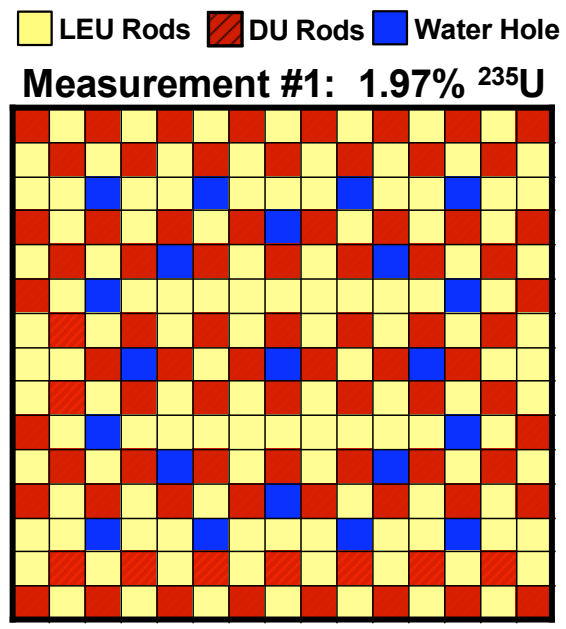

\begin{tabular}{|l|l|}
\hline Assembly Data \\
\hline Lattice geometry & $15 \times 15$ (square) \\
\hline Assembly width & $21 \mathrm{~cm}$ \\
\hline Fuel rod pitch & $1.4 \mathrm{~cm}$ \\
\hline Number of fuel rods & 204 \\
\hline Number of open channels & 21 \\
\hline Fuel Rod Data \\
\hline Fuel material & $\mathrm{UO}_{2}$ \\
\hline Cladding material & Zircaloy-2 \\
\hline Fuel pellet density & $10.48 \mathrm{~g} / \mathrm{cm}^{3}$ \\
\hline Fuel pellet diameter & $0.905 \mathrm{~cm}$ \\
\hline Outer rod diameter & $1.08 \mathrm{~cm}$ \\
\hline Cladding thickness & $0.095 \mathrm{~cm}$ \\
\hline Active fuel height & $103.5 \mathrm{~cm}$ \\
\hline
\end{tabular}

Fig. 4. Fuel rod configuration for measurement \#1 and specifications for LANL fuel assembly.

182 Prior to beginning the fresh fuel measurements, the gain setting was matched for all 4 pre183 amplifiers and tested with the fission chambers to ensure all detectors were working properly.

184 Standard ${ }^{235}$ U FCs from GE Reuter-Stokes [13] were used in SINRD. These FCs have a 5" active 
185 length and ${ }^{235} \mathrm{U}$ loading of $1.5-\mathrm{mg} / \mathrm{cm}^{2}$ (93 wt $\%{ }^{235} \mathrm{U}$ metal; $\left.0.13 \mathrm{~g}\right)$. Fig. 5 shows the inside of 186 the SINRD unit used for experimental measurements.
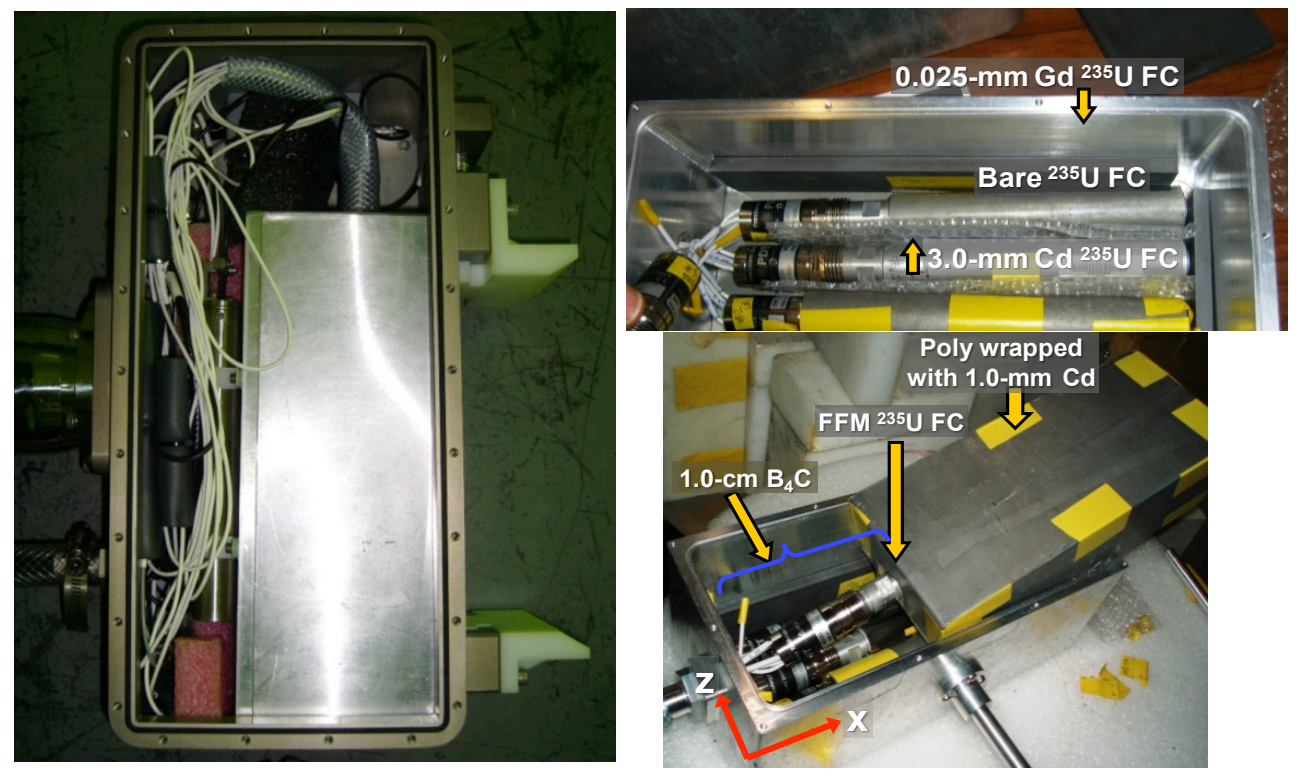

Fig. 5. Inside of the SINRD unit used for experimental measurements.

191 To minimize the probability of introducing systematic errors in our measurements from changes

192 in the geometry, the entire experiment was completely contained inside the water tank. A picture

193 of the SINRD experimental setup is shown in Fig. 6. In this experiment, the water surrounding

194 the fuel assembly moderates and reflects neutrons back into assembly and thus establishes a

195 neutron slowing-down energy spectrum in the fuel assembly. This moderated neutron spectrum 196 is especially important to achieving good counting statistics in the $3.0-\mathrm{mm} \mathrm{Cd}$ covered ${ }^{235} \mathrm{U}$ 197 fission chamber. The ${ }^{252} \mathrm{Cf}$ neutron source is placed in the center of the fuel assembly. It was 198 important that the axial location of the ${ }^{252} \mathrm{Cf}$ source is lined up with the center of the SINRD pod.

200 The singles rates from each fission chamber were recorded by splitting the signals between a List 201 Mode Multiplicity Module (LMMM) [14] and a JSR-15 with fast accidentals [15]. Both data 202 acquisition systems are used to count and record the total number of pulses and the time 203 correlation of coincidence pulses. The JSR-15 was connected to the laptop and IAEA Neutron 204 Coincidence Counting (INCC) software [16] version 5.1.2 was used to set the operating 205 parameters for SINRD, as well as collect and archive the measurement data. The LMMM was 
206 also connected to the laptop and the DAQ software was used to collect and archive the list mode 207 measurement data. A ${ }^{252} \mathrm{Cf}$ source with measured source strength of $\sim 1.0 \times 10^{6} \mathrm{n} / \mathrm{s}$ was placed 208 near the SINRD detector unit to ensure all of the FCs were working properly. The average count 209 time per measurement was approximately 3 hours.

Fig. 6. Picture of the experimental setup for SINRD measurements.

\section{3.2. Analysis of Results}

215 In order to observe how the SINRD and PNMC signatures change as a function of ${ }^{235} \mathrm{U}$, the 216 effective enrichment of PWR fuel assembly was varied from $1.97 \%$ to $0.22 \%{ }^{235} \mathrm{U}$ by uniformly 217 removing LEU fuel rods $\left(3.19 \%{ }^{235} \mathrm{U}\right)$ and replacing them with DU rods $\left(0.22 \%{ }^{235} \mathrm{U}\right)$ as shown 218 in Fig. 7. Since the detector can be applied to any four sides of the assembly, 4-quadrant 219 symmetry was assumed in fuel pin removal. In addition, the sensitivity of the SINRD and PNMC 220 signatures to a water gap was assessed by inserting $2.5 \mathrm{~mm}, 5 \mathrm{~mm}$, and $7.5 \mathrm{~mm}$ thick aluminum 221 inserts between the detector and the fuel assembly for each measurement configuration. The 222 aluminum inserts were only $2 \mathrm{~cm}$ wide and placed in front of the preamplifiers so as not to 223 impact the water gap between the detector and fuel assembly. 
$\square$ LEU Rods $\square$ DU Rods $\quad$ Replace with DU $\square$ Water Hole

Measurement \#1: 1.97\% ${ }^{235} \mathrm{U}$ Measurement \#2: 1.44\% ${ }^{235 \mathrm{U}}$ Measurement \#3: $0.80 \%{ }^{235 \mathrm{U}}$ Measurement \#4: $0.22 \%{ }^{235 \mathrm{U}}$ 120 LEU Rods, 84 DU Rods 84 LEU Rods, 120 DU Rods 40 LEU Rods, 164 DU Rods 0 LEU Rods, 204 DU Rods


Fig. 7. Fuel rod configurations for fresh fuel measurements.

227 The measured count rates and relative uncertainties from the SINRD FCs are given in Table 1.

228 The relative standard deviation (RSD) of the measured count rates for each water gap case per

229 effective ${ }^{235} \mathrm{U}$ enrichment is also given in Table 1.

Table 1. Measured count rates and relative uncertainties from SINRD FCs.

\begin{tabular}{|c|c|c|c|c|c|c|}
\hline \multirow{2}{*}{$\begin{array}{l}{ }^{235} \mathrm{U} \\
\text { Content }\end{array}$} & \multirow{2}{*}{$\begin{array}{l}\text { Count } \\
\text { Time }\end{array}$} & \multirow{2}{*}{$\begin{array}{c}\text { Water } \\
\text { Gap }\end{array}$} & \multicolumn{4}{|c|}{ Count Rates in SINRD FCs [cps] } \\
\hline & & & FFM $\left(B_{4} C\right)$ & Bare ${ }^{235} \mathrm{U}$ & $\mathbf{G d}^{235} \mathbf{U}$ & $C d^{235} \mathrm{U}$ \\
\hline \multirow{5}{*}{$\begin{array}{c}0.22 \% \\
(3.7 \mathrm{~g} / \mathrm{cm})\end{array}$} & $30 s \times 2058 c y c$ & $0.0 \mathrm{~mm}$ & $52.55 \pm 0.06 \%$ & $47.27 \pm 0.06 \%$ & $7.419 \pm 0.15 \%$ & $2.073 \pm 0.28 \%$ \\
\hline & $30 s \times 208 c y c$ & $2.5 \mathrm{~mm}$ & $49.19 \pm 0.18 \%$ & $45.69 \pm 0.19 \%$ & $7.038 \pm 0.48 \%$ & $1.916 \pm 0.91 \%$ \\
\hline & $30 \mathrm{~s} \times 275$ cyc & $5.0 \mathrm{~mm}$ & $47.46 \pm 0.16 \%$ & $43.52 \pm 0.17 \%$ & $6.788 \pm 0.42 \%$ & $1.868 \pm 0.80 \%$ \\
\hline & $30 s \times 276 c y c$ & $7.5 \mathrm{~mm}$ & $45.32 \pm 0.16 \%$ & $43.50 \pm 0.17 \%$ & $6.694 \pm 0.42 \%$ & $1.753 \pm 0.83 \%$ \\
\hline & & RSD = & $6.28 \%$ & 4.08\% & 4.64\% & $6.98 \%$ \\
\hline \multirow{4}{*}{$\begin{array}{c}\mathbf{0 . 8 0 \%} \\
(10.6 \mathrm{~g} / \mathrm{cm})\end{array}$} & 30 s $\times 2084$ cyc & $0.0 \mathrm{~mm}$ & $79.15 \pm 0.04 \%$ & $50.34 \pm 0.06 \%$ & $9.210 \pm 0.13 \%$ & $2.897 \pm 0.23 \%$ \\
\hline & $30 \mathrm{~s} \times 360$ cyc & $5.0 \mathrm{~mm}$ & $77.54 \pm 0.11 \%$ & $46.93 \pm 0.14 \%$ & $8.930 \pm 0.32 \%$ & $2.643 \pm 0.59 \%$ \\
\hline & $30 s \times 200$ cyc & $7.5 \mathrm{~mm}$ & $71.12 \pm 0.15 \%$ & $48.01 \pm 0.19 \%$ & $8.661 \pm 0.44 \%$ & $2.616 \pm 0.80 \%$ \\
\hline & & RSD = & $5.59 \%$ & $3.61 \%$ & $3.07 \%$ & $5.70 \%$ \\
\hline \multirow{3}{*}{$\begin{array}{c}1.44 \% \\
(18.1 \mathrm{~g} / \mathrm{cm})\end{array}$} & 30s x 1948cyc & $0.0 \mathrm{~mm}$ & $121.5 \pm 0.04 \%$ & $50.87 \pm 0.06 \%$ & $11.56 \pm 0.12 \%$ & $4.059 \pm 0.21 \%$ \\
\hline & $30 \mathrm{~s} \times 340$ cyc & $5.0 \mathrm{~mm}$ & $112.6 \pm 0.09 \%$ & $47.63 \pm 0.14 \%$ & $10.82 \pm 0.30 \%$ & $3.716 \pm 0.51 \%$ \\
\hline & & RSD = & $4.46 \%$ & $3.33 \%$ & $3.44 \%$ & $4.60 \%$ \\
\hline \multirow{5}{*}{$\begin{array}{c}1.97 \% \\
(24.3 \mathrm{~g} / \mathrm{cm})\end{array}$} & 30s x 2090cyc & $0.0 \mathrm{~mm}$ & $143.6 \pm 0.03 \%$ & $50.54 \pm 0.06 \%$ & $13.07 \pm 0.11 \%$ & $3.808 \pm 0.51 \%$ \\
\hline & $30 \mathrm{~s} \times 540$ cyc & $2.5 \mathrm{~mm}$ & $141.8 \pm 0.07 \%$ & $49.93 \pm 0.11 \%$ & $12.38 \pm 0.22 \%$ & $4.806 \pm 0.18 \%$ \\
\hline & 30 s x 2880cyc & $5.0 \mathrm{~mm}$ & $139.8 \pm 0.03 \%$ & $51.16 \pm 0.05 \%$ & $12.59 \pm 0.10 \%$ & $4.563 \pm 0.37 \%$ \\
\hline & 30s x 497cyc & $7.5 \mathrm{~mm}$ & $123.4 \pm 0.07 \%$ & $58.82 \pm 0.11 \%$ & $12.81 \pm 0.23 \%$ & $4.647 \pm 0.16 \%$ \\
\hline & & RSD = & $6.80 \%$ & $7.93 \%$ & $2.35 \%$ & $2.51 \%$ \\
\hline
\end{tabular}


233 The results for the measured FFM/TFM, FFM/(Gd-Cd), FFM/Gd and FFM/Cd ${ }^{235} \mathrm{U}$ FC ratios

234 with no water gap are shown in Fig. 8 versus the effective ${ }^{235} \mathrm{U}$ enrichment in the FA. These 235 results are normalized to the case with all DU fuel rods. Based on these results, we see that the 236 slope of the FFM/TFM ratio (PNMC signature) is $\sim 45 \%$ greater than the SINRD signature ratios. 237 The fresh fuel measurements are advantageous because the FFM / TFM ratio is not complicated 238 by the presence of fission product absorbers which also impact the neutron leakage 239 multiplication. It should also be noted that the $1 \sigma$ error bars are smaller than the data symbols.



Fig. 8. Normalized SINRD-PNMC ratios versus ${ }^{235} \mathrm{U}$ enrichment for no water gap case.

244 Fig. 9 shows the normalized (a) FFM / TFM and (b) FFM / (Gd-Cd) ${ }^{235} \mathrm{U}$ FC ratios versus ${ }^{235} \mathrm{U}$ 245 enrichment for different water gaps. MCNP results are shown for the $0 \mathrm{~mm}$ WG case. The results 246 are normalized to the case with all DU fuel rods. These results show that both ratios are not 247 sensitive to water gaps of 5-mm or less. We find good agreement between the simulated and 248 experimental results. This is important because any bias in the MCNP results is expected to 249 mostly cancel out in the normalization. The $1 \sigma$ error bars are smaller than the data symbols. 


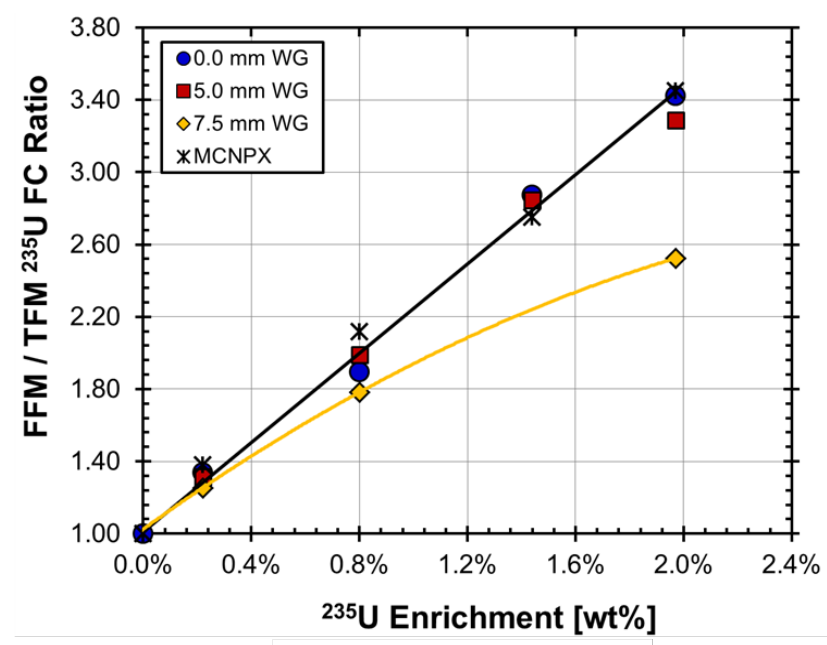

(a) FFM / TFM Ratio

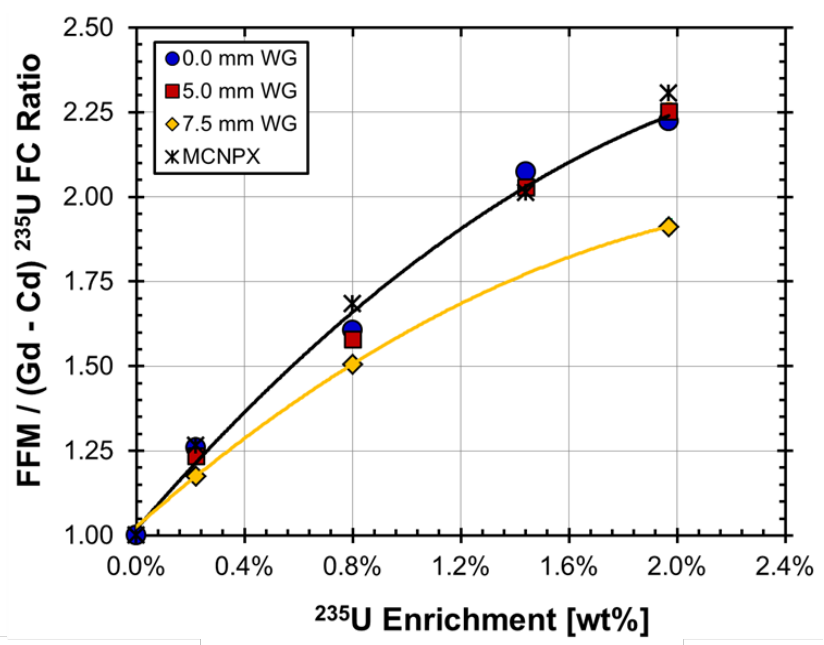

(b) FFM / (Gd - Cd) Ratio
Fig. 9. Normalized (a) FFM / TFM and (b) FFM / (Gd-Cd) ${ }^{235} \mathrm{U} F C$ ratios versus ${ }^{235} \mathrm{U}$ enrichment for different water gaps. MCNP results are shown for the $0 \mathrm{~mm}$ WG case.

The MCNP code calculates the expected fission rate in each of the four SINRD FCs. However, the absolute accuracy of the calculations is limited by the lack of knowledge of the ${ }^{235} \mathrm{U}$ deposit in the FCs such as its depth and position within the FCs, and the actual thicknesses of Gd and Cd filters. In the simulations, the nominal dimensions obtained from the vendor were used. We did not account for the non-uniformities in the $\mathrm{Gd}$ and $\mathrm{Cd}$ filter thicknesses. Additional potential sources of bias that were not accounted for in the MCNP simulations include: location and geometry of the ${ }^{252} \mathrm{Cf}$ source (modeled as a point source), energy spectrum of the ${ }^{252} \mathrm{Cf}$ source, location of the FCs within the SINRD pod, room return neutrons, and efficiency of the SINRD FCs (e.g. no counts are thrown away in MCNP).

To remove these sources of bias from the MCNP results, we have normalized the SINRD ratios to a standard calibration fuel assembly. For this experiment, the SINRD FC ratios were normalized to the DU fuel assembly. Fig. 10 shows the normalized Calculated to Experimental ratio (C/E ratio) versus effective ${ }^{235} \mathrm{U}$ enrichment for different SINRD ratios. The results are relatively constant for all cases. For the normalized $\mathrm{C} / \mathrm{E}$ ratios, the average RSD was $\sim 3.7 \%$. This confirms that any bias in the MCNP results cancels out in the normalization. Thus, in order to ensure our SINRD FC ratios are insensitive to any potential sources of bias in the MCNP results or measurements, SINRD requires calibration with a reference assembly of similar geometry. 


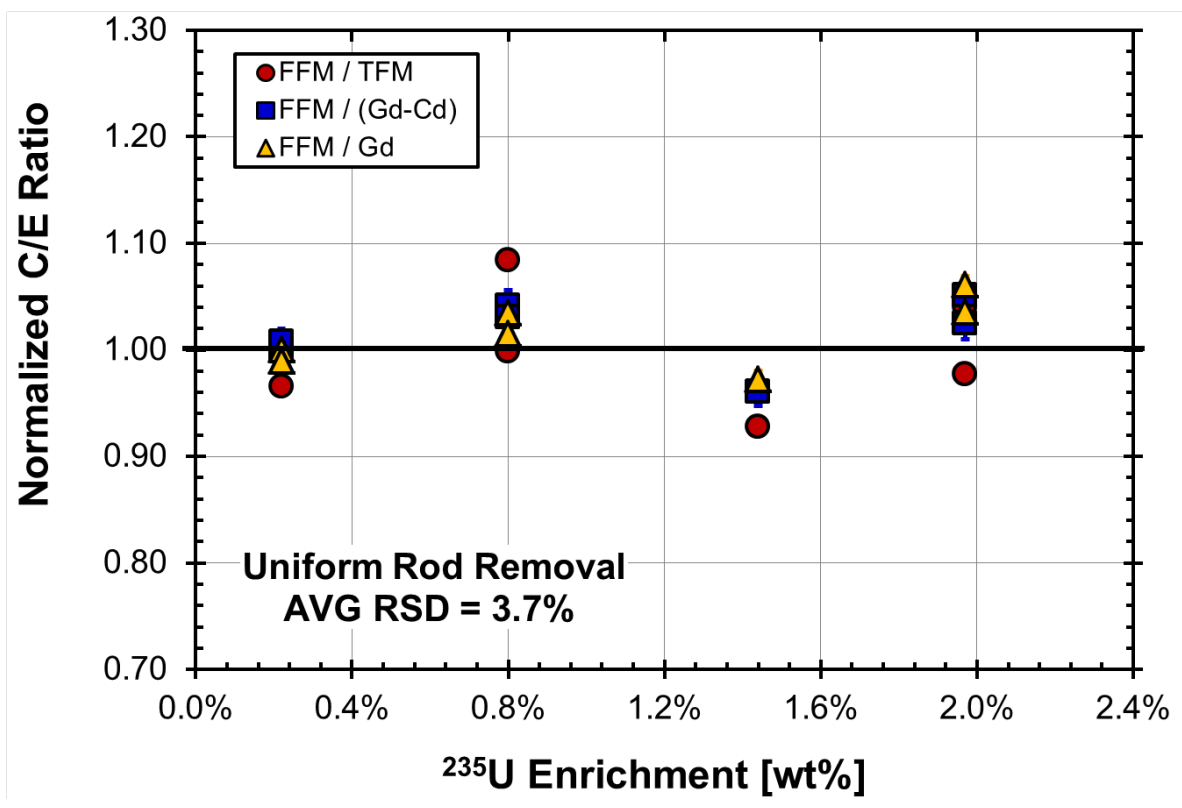

Fig. 10. Normalized $\mathrm{C} / \mathrm{E}$ ratio versus ${ }^{235} \mathrm{U}$ enrichment.

\section{Spent Fuel Measurements in ROK}

278 Measurements of PWR spent fuel assemblies (SFAs) were performed with the SINRD-PNMC 279 detector at KAERI's PIEF in ROK. It is important to note that the same detector used for the 280 fresh fuel measurements described above was used for the spent fuel measurements in ROK.

\subsection{Overview of Spent Fuel Measurements}

283 Four different PWR SFAs shown in Fig. 11 were measured with the SINRD-PNMC detector and 284 of these SFAs, FA3 was the only SFA not in a shroud and with no fuel rods removed. Prior to 285 beginning the measurements, the SFA was lifted out of the storage rack and then the detector 286 was moved to the SFA. For each SFA, 3 different positions were measured along the burnup 287 gradient on all 4 sides of the SFA. The approximate count time per measurement was 8 minutes. 288 In addition, several positions on the burnup plateau were measured multiple times to assess the 289 measurement reproducibility and systematic errors of the SINRD detector.

291 It is important to note that FA 1, 6, and 7 were in a shroud with variable water gap and fuel rod 292 removal (only significant effect for FA7) on each side which complicated analysis of the results 
293 because both of these variables impact the neutron multiplication (PNMC signature) and the 294 SINRD resonance signature.
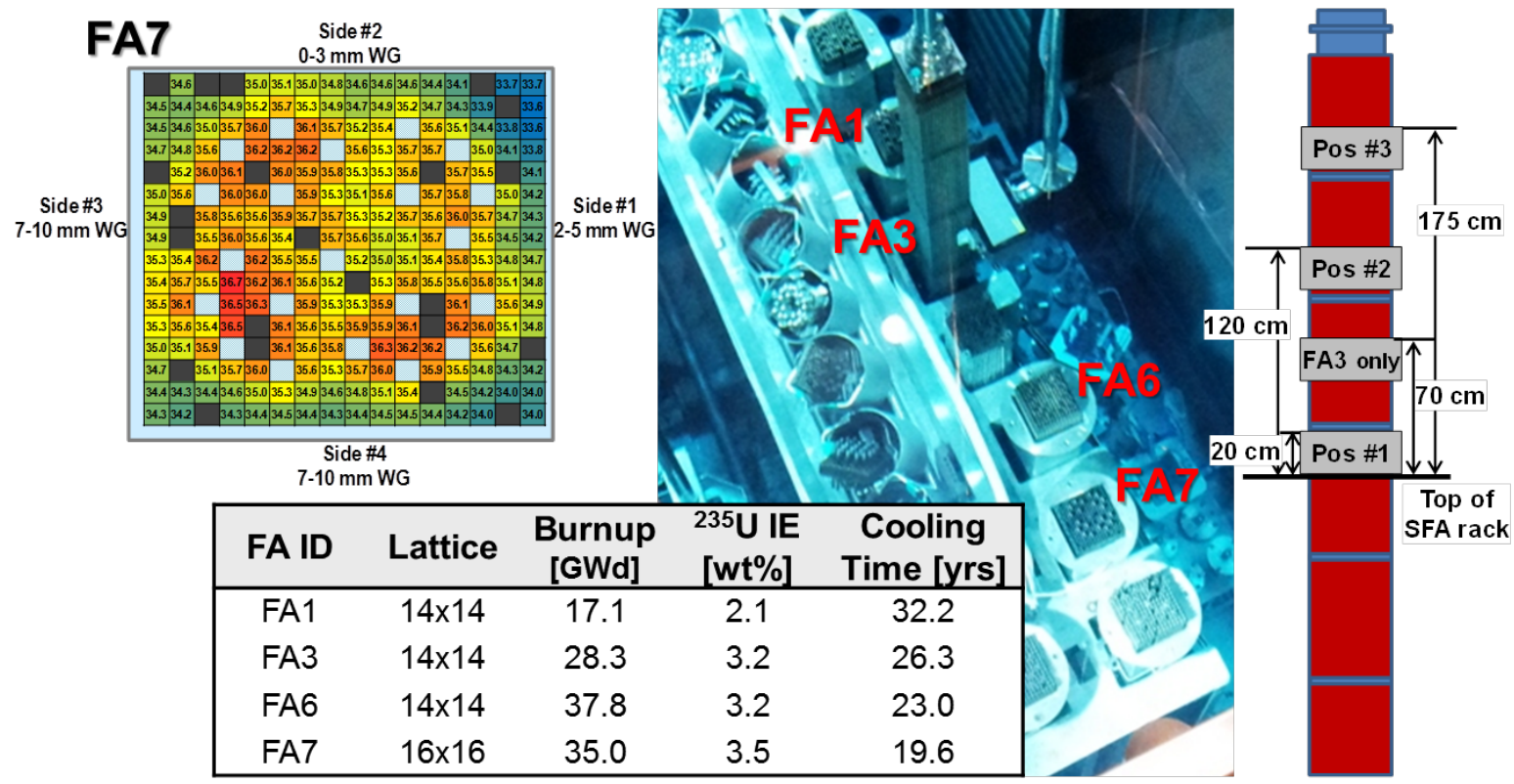

Fig. 11. PWR SFAs measured in ROK.

\subsection{Analysis of Results}

300 The gross gamma rate was used to confirm the axial location of SINRD on the SFAs both for 301 FA3 (no shroud) and for FA1, 6, and 7 located in shrouds. Fig. 12 shows the measured gross 302 gamma rate versus axial position for all SFAs and normalized gamma rate relative to fuel rod 303 scan for FA3 and FA6. Based on these results, we see that the top position for FA3 (no shroud) is 304 too close to the top of the SFA such that end effects (structural material at the ends of fuel rods) 305 dominate the measured count rates and are not representative of what we would expect to see 306 from a lower burnup. As a result, this data point was not used in the analysis of results for FA3. 307 In general, it was difficult to position the detector close enough to the top of the SFA to see a 308 change in burnup but not too close to the top such that end effects dominated the measured 309 signal. 

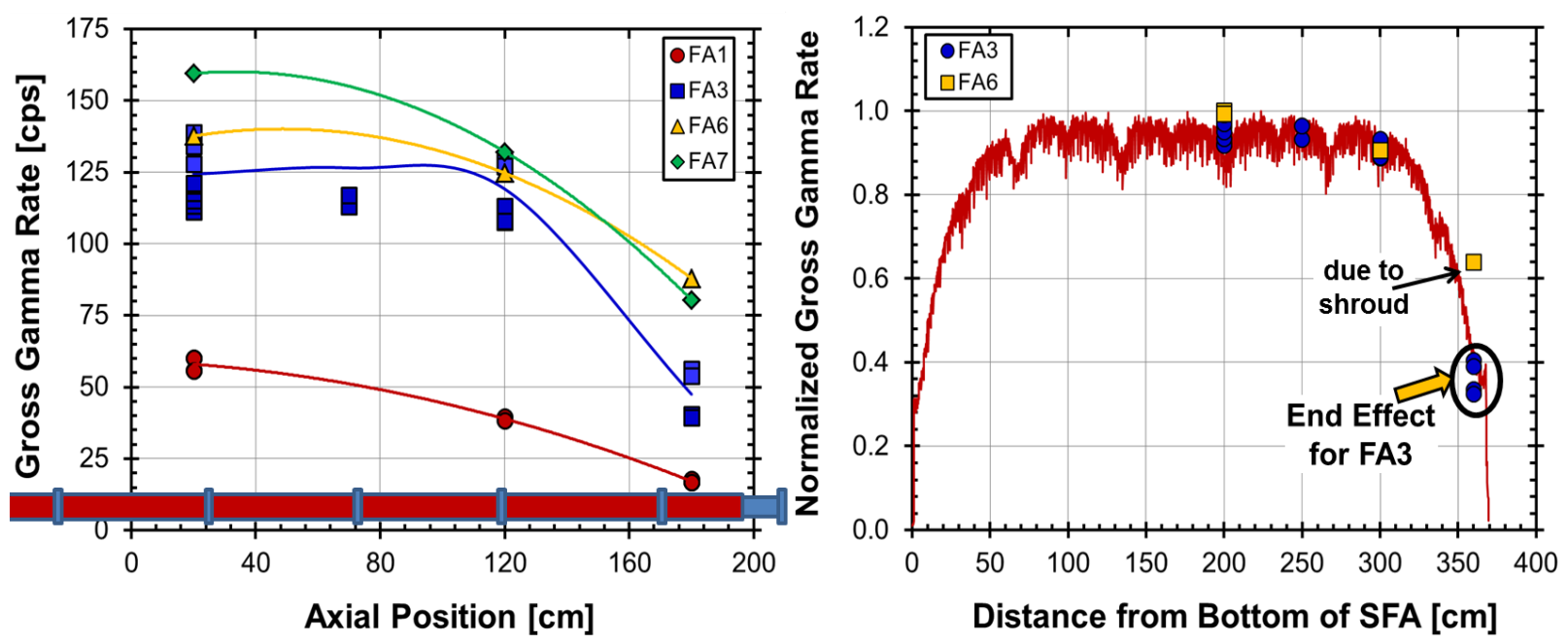

Fig. 12. Gross gamma rate versus axial position for all measured SFAs and Normalized Gamma rate relative to fuel rod scan for FA3 and FA6.

316 Fig. 13 shows the PNMC FFM/TFM ratio versus axial position and burnup for FA 1, 3, 6, 7 on

317 the side of the SFA with minimum water gap (except FA3 which had no water gap). These 318 measurements were performed at different axial positions and the burnup was estimated based 319 gamma scans of the fuel assemblies. The overall change in the FFM/TFM ratio is relatively small 320 for a given fuel assembly which may be attributed to the small change in burnup as a function of 321 axial position. However, the results are consistent with the change in neutron multiplication we 322 would expect from the different burnups of the SFAs and we see a distinct difference in the 323 ratios for the different burnups. It is important to note that the FFM / TFM ratio is complicated 324 by the presence of fission product absorbers which also impact the neutron leakage 325 multiplication.

327 For FA3 (no shroud), we attempted to introduce a 5-7 mm water gap between the SFA and 328 SINRD by mechanically moving SINRD back $1 / 2$ to $3 / 4$ of a turn. The thickness of the water gap 329 was a best estimate to $\pm 50 \%$ based on the distance we turned the lever and was further 330 complicated by the swaying of the SFA in the water. Fig. 14 shows the FFM / TFM ratio versus 331 FA3 SFA side for different water gaps. The FFM / TFM ratio changed by $4.6 \%$ for $\sim 5-\mathrm{mm}$ water 332 gap and by $10.8 \%$ (average) for the $\sim 7-\mathrm{mm}$ water gap case. It is important to note that the results 333 are consistent for all 4 sides of the FA3 SFA. 


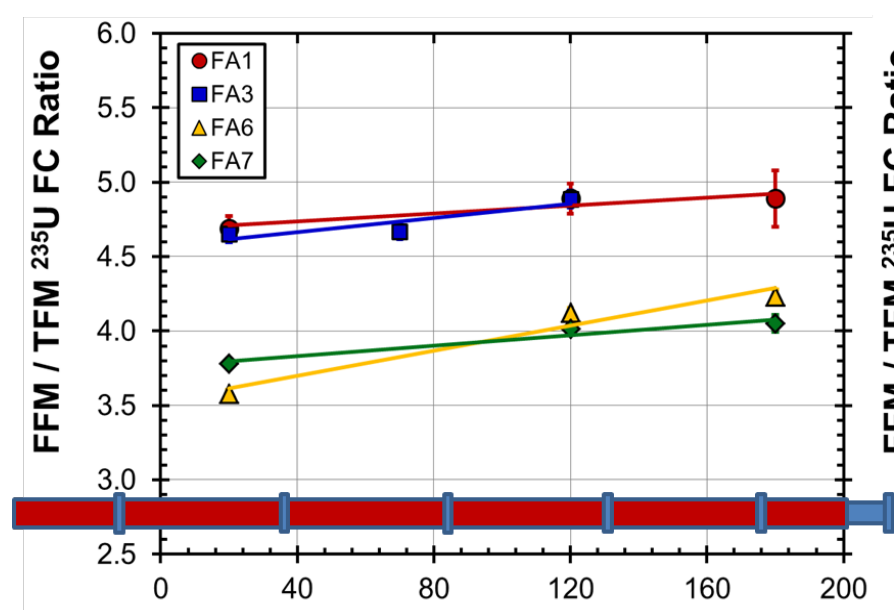

Axial Position [cm]

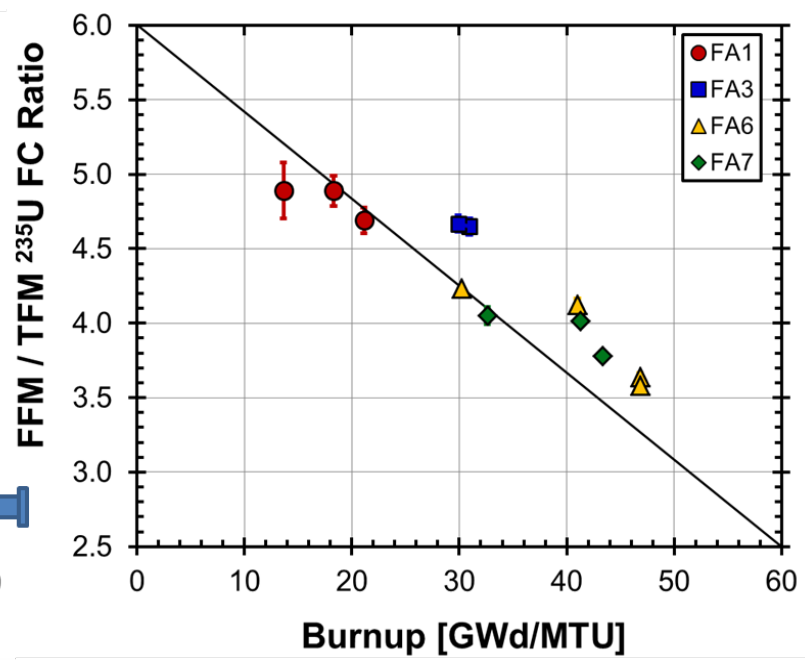

Fig. 13. FFM / TFM ratio versus axial position and burnup for FA 1, 3, 6, 7 on the side of the SFA with minimum water gap.
336

337

338

346 signature.

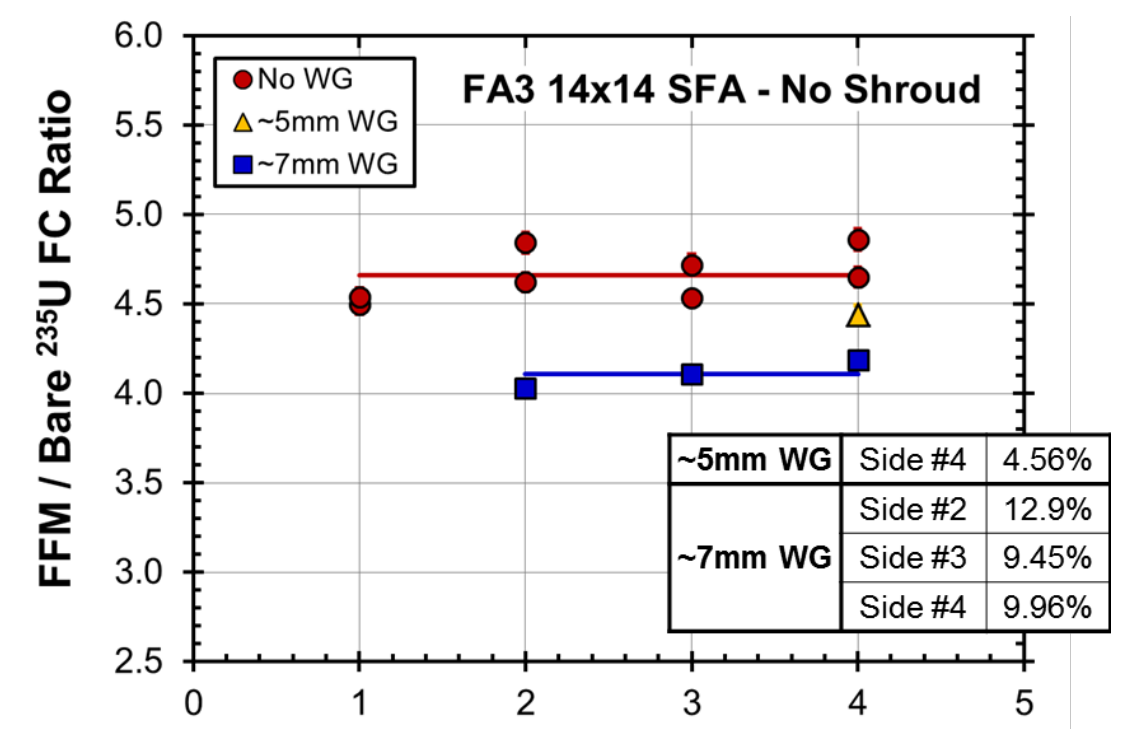

FA3 SFA Side

Fig. 14. FFM / TFM ratio versus FA3 SFA side for different water gaps.

The change in SINRD FC ratios was also analyzed for the different measured SFAs. However, it is important to note that when measuring near the top of the SFA (i.e. burnup gradient) each of the SINRD FCs saw a different burnup and thus fissile content. Therefore, plotting the SINRD ratios versus fissile content calculated based on axial position is not a valid test of the SINRD 


\subsection{Uncertainty Analysis}

349 The measured count rates $\pm 1 \sigma$ and average rate \pm RSD for each side of FA3 (no shroud) at axial 350 distance of $20 \mathrm{~cm}$ from top of SFA storage rack are given in Table 2. For each of the SINRD 351 measurements, SINRD was lowered by crane to the axial position of $20 \mathrm{~cm}$ above the SFA 352 storage rack and moved towards the SFA until contact was made to ensure the water gap 353 between the detector and fuel assembly was essentially zero. The axial position for these 354 measurements was close to the center of the SFA in the plateau region of the burnup profile such 355 that the uncertainty in the axial position (i.e. $20 \mathrm{~cm} \pm 2 \mathrm{~cm}$ ) was negligible. These results show 356 that the measured count rates are reproducible and consistent with the radial burnup gradient 357 across FA3. In addition, the random and systematic uncertainties were less than 5\% for all of the 358 measured rates for all sides of FA3 except Side \#3. The variability in the count rates for Side \#3 359 may be attributed to movement of the SFA and/or detector during the measurement which would 360 have been most likely to occur during the $\sim 2.6$ hour long measurement which was only taken on 361 Side \#3. Practical implementation of the SINRD for verification of spent fuel would be in a Fork 362 detector [17]. The two-sided measurement of the Fork would further reduce systematic 363 uncertainties from positioning and unknown water gap.

Table 2. Measured count rates $\pm 1 \sigma$ and average rate \pm RSD for each side at axial distance of $20 \mathrm{~cm}$ from 366 top of SFA storage rack of FA3 (no shroud).

\begin{tabular}{|c|c|c|c|c|c|c|}
\hline \multirow{2}{*}{$\begin{array}{c}\text { FA3 } \\
\text { (No Shroud) }\end{array}$} & \multirow{2}{*}{$\begin{array}{l}\text { Count } \\
\text { Time [s] }\end{array}$} & \multicolumn{5}{|c|}{ Measured Count Rates $\pm 1 \sigma$ [cps] } \\
\hline & & $\mathrm{ia}$ & FFM & TFM & Gd FC & $\mathrm{Cd}$ \\
\hline \multirow{3}{*}{$\begin{array}{c}\text { Side \#1 } \\
\sim 30.1 \mathrm{GWd}\end{array}$} & & & $92.7 \pm 0.46 \%$ & $20.6 \pm 0.98 \%$ & $3.46 \pm 2.38 \%$ & $2.22 \pm$ \\
\hline & 520 & $139 \pm 0.37 \%$ & $92.5 \pm 0.46 \%$ & $20.4 \pm 0.97 \%$ & $3.46 \pm 2.36 \%$ & $2.14 \pm 3.00 \%$ \\
\hline & $A$ & $139 \pm 0.05 \%$ & $92.6 \pm 0.13 \%$ & & $3.46 \pm 0.10 \%$ & $8 \pm 2.44 \%$ \\
\hline \multirow{3}{*}{$\begin{array}{c}\text { Side \#2 } \\
\sim 29.6 \text { GWd }\end{array}$} & & & 8 & & & \\
\hline & 560 & $137 \pm$ & $87.7 \pm 0.45 \%$ & $18.1 \pm 0.99 \%$ & $3.25 \pm 2.34 \%$ & $1.92 \pm 3.05 \%$ \\
\hline & Avg \pm RSD $=$ & $136 \pm 1.61 \%$ & $86.6 \pm 1.79 \%$ & $18.3 \pm 1.50 \%$ & $3.16 \pm 4.14 \%$ & $1.94 \pm 1.30 \%$ \\
\hline \multirow{3}{*}{$\begin{array}{c}\text { Side \#3 } \\
\sim 25.6 \mathrm{GWd}\end{array}$} & $9<04$ & $113 \pm 0.10 \%$ & $70.5 \pm 0.12 \%$ & $15.5 \pm 0.26 \%$ & $2.56 \pm 0.65 \%$ & $1.53 \pm$ \\
\hline & 580 & $117 \pm 0.38 \%$ & $74.9 \pm 0.48 \%$ & $15.9 \pm 1.04 \%$ & $2.80 \pm 2.48 \%$ & $1.67 \pm 3.22 \%$ \\
\hline & Avg \pm RSD $=$ & $115 \pm 2.05 \%$ & $72.7 \pm 4.26 \%$ & $15.7 \pm 1.45 \%$ & $2.68 \pm 6.51 \%$ & $.92 \%$ \\
\hline \multirow{3}{*}{$\begin{array}{c}\text { Side \#4 } \\
\sim 26.7 \text { GWd }\end{array}$} & 519 & $120 \pm 0.40 \%$ & $80.0 \pm 0.49 \%$ & $16.5 \pm 1.08 \%$ & $2.74 \pm 2.65 \%$ & $1.67 \pm 3.40 \%$ \\
\hline & 516 & $121 \pm 0.40 \%$ & $79.3 \pm 0.49 \%$ & $17.1 \pm 1.07 \%$ & $2.81 \pm 2.63 \%$ & $1.73 \pm 3.35 \%$ \\
\hline & Avg \pm RSD $=$ & $121 \pm 0.57 \%$ & $79.7 \pm 0.60 \%$ & $16.8 \pm 2.48 \%$ & $2.77 \pm 1.65 \%$ & $1.70 \pm 2.50 \%$ \\
\hline
\end{tabular}


Table 3 shows the measured SINRD and PNMC ratios $\pm 1 \sigma$ RSD at axial distance of $20 \mathrm{~cm}$ from top of SFA storage rack for each side of FA3 (no shroud). Based on these results, we see that the RSD in the measurement is less than the random uncertainty (counting statistics) for most of the SINRD and PNMC ratios.

Table 3. SINRD and PNMC ratios $\pm 1 \sigma$ and average \pm RSD at axial distance of $20 \mathrm{~cm}$ from top of SFA storage rack for each side of FA3 (no shroud).

\begin{tabular}{|c|c|c|c|c|c|c|}
\hline $\begin{array}{c}\text { FA3 } \\
\text { (No Shroud) }\end{array}$ & $\begin{array}{c}\text { Count } \\
\text { Time [s] }\end{array}$ & FFM / TFM & $\begin{array}{c}\text { FFM I } \\
\text { (Gd-Cd) }\end{array}$ & FFM / Gd & FFM / Cd & $\begin{array}{c}\text { TFM / } \\
\text { (Gd-Cd) }\end{array}$ \\
\hline \multirow{3}{*}{$\begin{array}{c}\text { Side \#1 } \\
\sim 30.1 \mathrm{GWd}\end{array}$} & 510 & $.08 \%$ & $74.3 \pm 8.48 \%$ & $26.8 \pm 2.42 \%$ & $41.8 \pm 3.01 \%$ & $16.5 \pm 8.52 \%$ \\
\hline & 520 & $4.54 \pm 1.07 \%$ & $70.2 \pm 7.89 \%$ & $26.7 \pm 2.40 \%$ & $43.2 \pm 3.03 \%$ & $15.5 \pm 7.94 \%$ \\
\hline & Avg \pm RSD = & $4.52 \pm 0.68 \%$ & $72.3 \pm 4.00 \%$ & $26.7 \pm 0.02 \%$ & $42.5 \pm 2.32 \%$ & $16.0 \pm 4.68 \%$ \\
\hline \multirow{3}{*}{$\begin{array}{c}\text { Side \#2 } \\
\sim 29.6 \mathrm{GWd}\end{array}$} & 581 & $4.62 \pm 1.06 \%$ & $76.9 \pm 8.37 \%$ & $27.9 \pm 2.41 \%$ & $43.7 \pm 3.00 \%$ & $16.6 \pm 8.42 \%$ \\
\hline & 560 & $4.84 \pm 1.09 \%$ & $65.8 \pm 7.23 \%$ & $27.0 \pm 2.39 \%$ & $45.7 \pm 3.08 \%$ & $13.6 \pm 7.28 \%$ \\
\hline & Avg \pm RSD = & $4.73 \pm 3.28 \%$ & $71.4 \pm 11.0 \%$ & $27.4 \pm 2.35 \%$ & $44.7 \pm 3.08 \%$ & $15.1 \pm 14.2 \%$ \\
\hline \multirow{3}{*}{$\begin{array}{c}\text { Side \#3 } \\
\sim 25.6 \mathrm{GWd}\end{array}$} & 9264 & $4.54 \pm 0.29 \%$ & $68.8 \pm 2.05 \%$ & $27.6 \pm 0.66 \%$ & $46.0 \pm 0.85 \%$ & $15.2 \pm 2.07 \%$ \\
\hline & 580 & $4.72 \pm 1.15 \%$ & $65.8 \pm 7.73 \%$ & $26.7 \pm 2.53 \%$ & $44.9 \pm 3.25 \%$ & $13.9 \pm 7.79 \%$ \\
\hline & Avg \pm RSD = & $4.63 \pm 2.82 \%$ & $67.3 \pm 3.13 \%$ & $27.1 \pm 2.25 \%$ & $45.5 \pm 1.66 \%$ & $14.6 \pm 5.95 \%$ \\
\hline \multirow{3}{*}{$\begin{array}{c}\text { Side \#4 } \\
\sim 26.7 \text { GWd }\end{array}$} & 519 & $4.86 \pm 1.19 \%$ & $74.5 \pm 8.60 \%$ & $29.2 \pm 2.70 \%$ & $48.0 \pm 3.44 \%$ & $15.3 \pm 8.65 \%$ \\
\hline & 516 & $4.65 \pm 1.17 \%$ & $73.5 \pm 8.70 \%$ & $28.3 \pm 2.67 \%$ & $45.9 \pm 3.38 \%$ & $15.8 \pm 8.75 \%$ \\
\hline & Avg \pm RSD $=$ & $4.75 \pm 3.09 \%$ & $74.0 \pm 0.93 \%$ & $28.7 \pm 2.26 \%$ & $46.9 \pm 3.10 \%$ & $15.6 \pm 2.16 \%$ \\
\hline
\end{tabular}

\subsection{Sensitivity to Partial Defects}

The sensitivity and penetrability of SINRD was assessed by modeling partial defects in PWR $17 \times 17$ spent LEU fuel assemblies with an initial enrichment of $4 \%{ }^{235} \mathrm{U}$. We removed 40 fuel rods from the center of the assembly and 20 and 40 fuel rods uniformly from the fuel assembly. The rods were replaced with either DU or NU rods or left void (filled with water). The percent change in the FFM / TFM ratio was calculated for each case to determine if the diverted rods can be detected with a $3 \sigma$ confidence level. A count time of 12 minutes was assumed. Fig. 15(a) shows the FFM / TFM ratio versus neutron multiplication and Fig. 15(b) shows the FFM count rate versus FFM / TFM ratio for the cases with no defects and partial defects. These results show that all of the uniform partial defects cases can be detected with a $3 \sigma$ confidence level but only 1 of the cases with fuel rods removed from the center of the fuel assembly. It should also be noted that the cases with center partial defects have a higher ratio than the no defects case whereas the cases with uniform partial defects have a lower ratio. A customized removal of rods from the 
390

center and sides of SFA could reduce the sensitivity to the removal; however, the gross neutron rate in the FFM ${ }^{235} \mathrm{U} F C$ would be perturbed as shown in Fig. 15(b) indicating an anomaly.
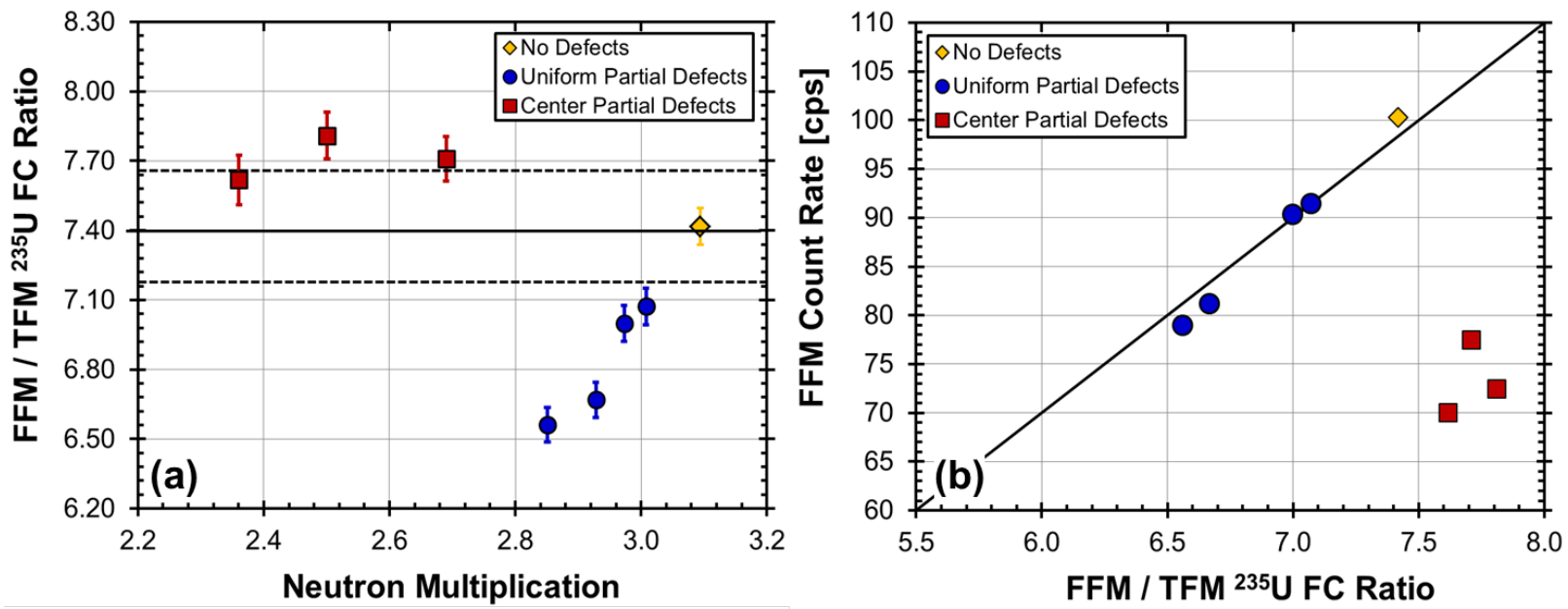

Fig. 15. (a) FFM/TFM ratio versus neutron multiplication and (b) FFM count rate versus FFM/TFM ratio for the cases with no defects and partial defects.

\section{Conclusions}

SINRD is a new NDA technique that was developed at LANL to improve existing nuclear safeguards measurements for LWR fuel assemblies. It is important to note that two different techniques can be utilized using the same SINRD detector and hardware:

- PNMC Method: uses the ratio of the FFM to TFM to quantify the neutron leakage multiplication of the item

- SINRD Method: uses the Gd and $\mathrm{Cd}$ covered ${ }^{235} \mathrm{U}$ FCs to measure the resonance absorption from ${ }^{235} \mathrm{U}$ and ${ }^{239} \mathrm{Pu}$ in spent fuel.

We have performed MCNP simulations and experimental measurements of fresh and spent PWR fuel assemblies using the SINRD detector. The purpose of these experiments was to assess the following capabilities of SINRD: 1) reproducibility of measurements to quantify systematic errors, 2) sensitivity to water gap between detector and fuel assembly, 3) sensitivity and penetrability to the removal of fuel rods from the assembly, and 4) use of PNMC/SINRD ratios to quantify neutron multiplication and/or fissile content. It should also be noted that the SINRD detector requires a calibration with a reference assembly of similar geometry. However, since both SINRD and PNMC methods uses ratios of different FCs, we expect most of the systematic errors related to calibration and positioning cancel in the normalized ratio to the reference 
413 assembly. The results from these simulations and measurements provide valuable experimental 414 data that directly supports safeguards R\&D efforts on the viability of passive neutron NDA 415 techniques and detector designs for partial defect verification of spent fuel assemblies.

417 For the fresh fuel measurements, the effective ${ }^{235} \mathrm{U}$ enrichment of PWR fuel assembly was varied 418 from $1.97 \%$ to $0.22 \%{ }^{235} \mathrm{U}$ by uniformly removing LEU fuel rods $\left(3.19 \%{ }^{235} \mathrm{U}\right)$ and replacing 419 them with DU rods $\left(0.22 \%{ }^{235} \mathrm{U}\right)$. In addition, the sensitivity of the SINRD and PNMC signatures 420 to a water gap was assessed by inserting $2.5 \mathrm{~mm}, 5 \mathrm{~mm}$, and $7.5 \mathrm{~mm}$ thick aluminum inserts 421 between the detector and the fuel assembly for each measurement configuration. These results 422 show that both the SINRD and PNMC ratios were not sensitive to water gaps of 5-mm or less. 423 We find good agreement between the simulated and experimental results. This is important 424 because any bias in the MCNP results is expected to mostly cancel out in the normalization to 425 the reference assembly which in this case was the all DU rod FA.

427 For the spent fuel measurements, 4 different SFAs were measured at PIEF at KAERI. Three 428 different axial positions were measured along the burnup gradient on all 4 sides of the SFA 429 where the position near the center of the SFA was measured multiple times to assess systematic 430 uncertainties. The approximate count time per measurement was 8 minutes. For all of the SINRD 431 and PNMC ratios except the FFM / (Gd-Cd) ratio, the total measurement uncertainty was less 432 than 5\%. However, the total uncertainty in the FFM / (Gd-Cd) ratio could easily be reduced by 433 increasing the count time. It is important to note that FA 1, 6, and 7 were in a shroud with 434 variable water gap on each side and fuel rod removal (FA7 only) which complicated analysis of 435 the results because both of these variables impact the neutron multiplication and the SINRD 436 resonance signature. The overall change in the FFM/TFM ratio is relatively small for a given fuel 437 assembly which may be attributed to the small change in burnup as a function of axial position. 438 However, the results are consistent with the change in neutron multiplication we would expect 439 from the different burnups of the SFAs and we see a distinct difference in the ratios for the 440 different burnups. The change SINRD signature ratios were also analyzed for the different 441 measured SFAs. However, it is important to note that each of the SINRD FCs sees a different 442 burnup and thus fissile content when measuring near the top of the SFA (i.e. burnup gradient). 
443 Thus, plotting the SINRD ratios versus fissile content calculated based on axial position is not a 444 valid test of the SINRD signature.

446 Overall, SINRD is a relatively low-cost, robust hardware system that could be deployed for 447 verification of spent fuel assemblies. Practical implementation of SINRD for spent fuel 448 verification would be in Fork detector or with the CIPN detector given the complimentary nature 449 of the hardware. The two-sided measurement of the Fork would further improve counting 450 statistics and reduce systematic uncertainties from positioning and unknown water gap. This 451 enhanced Fork detector would provide information on the neutron energy leakage spectra that 452 would enhance the robustness of the spent fuel verification measurement.

\section{Acknowledgements}

455 We would like to acknowledge the Department of Energy National Nuclear Security 456 Administration's Office of Nonproliferation and International Security (NA-24) and the Program 457 of Technical Assistance (POTAS) to the IAEA for their support in the development of the 458 SINRD method. The IAEA has provided useful guidance and support for this research. This 459 work was funded under NA-241 Next Generation Safeguards Initiative (NGSI) and International 460 Nuclear Safeguards Engagement Program (INSEP).

\section{References}

[1] R. Hooper, The Changing Nature of Safeguards, International Atomic Energy Agency Bulletin Vol. 45, Issue 1, Vienna, Austria, June 2003.

[2] "Design Measures to Facilitate Implementation of Safeguards at Future Water Cooled Nuclear Power Plants," International Atomic Energy Agency Safeguards Technical Reports Series No. 392, Vienna, Austria (1998).

[3] M.T. Swinhoe, H.O. Menlove, D.H. Beddingfield, "A Survey of LWR Spent Fuel and Measurement Methods," Los Alamos National Laboratory Report, LA-UR-02-6996, August 2002.

[4] A.M. LaFleur, W.S. Charlton, H.O. Menlove, M.T. Swinhoe, Alain R. Lebrun, "Development of Self-Interrogation Neutron Resonance Densitometry to Improve 
Detection of Partial Defects in PWR Spent Fuel Assemblies," Nuclear Technology, 181 (2013) pp. 354-370.

[5] H.O. Menlove, C.D. Tesche, M.M. Thorpe, R.B. Walton, A Resonance Self-Indication Technique for Isotopic Assay of Fissile Materials, Nucl. Appl., 6 (1969).

[6] Nondestructive Assay of SEFOR Fuel Rods, Los Alamos National Laboratory Program Status Report April-June 1969, LA-4227-MS (1969).

[7] Neutron Self-Indication Assay of SEFOR Fuel Rods, Los Alamos National Laboratory Program Status Report July-September1969, LA-4315-MS (1969).

[8] A.M. LaFleur, W.S. Charlton, H.O. Menlove, M.T. Swinhoe, "Development of SelfInterrogation Neutron Resonance Densitometry to Quantify the Fissile Content in PWR Spent LEU and MOX Assemblies," Nuclear Science and Engineering, 171 (2012) pp.175-191.

[9] A.M. LaFleur, "Development of Self-Interrogation Neutron Resonance Densitometry (SINRD) to Measure the Fissile Content in Nuclear Fuel," Ph.D. Dissertation, Texas A\&M University, August 2011.

[10] Nuclear Energy Agency, JANIS 3.0 User's Guide, Organization for Economic Cooperation and Development, June 2007.

[11] A.M. LaFleur, W.S. Charlton, H.O. Menlove, M.T. Swinhoe, "Comparison of Fresh Fuel Experimental Measurements to MCNP Calculations Using Self-Interrogation Neutron Resonance Densitometry," Nuclear Instruments and Methods in Physics Research A, 680 (2012) 168-178.

[12] H.O. Menlove, J.E. Pieper, Neutron Collar Calibration for Assay of LWR Fuel Assemblies, Los Alamos National Laboratory Report, LA-10827-MS, March 1987.

[13] Reuter Stokes Fission Counter (RS-P6-0805-134) Fact Sheet, General Electric Company, GEA-13541B [http://www.ge-mcs.com/download/reuter-stokes/gea13541b.pdf], (2005).

[14] D. Henzlova, H.O. Menlove, M.T. Swinhoe, et al., "Neutron Data Collection and Analysis Techniques Comparison for Safeguards," IAEA Symposium, IAEA-CN-184/178, (2010).

[15] N. Menaa, M. Villani, S. Croft, B. McElroy, et. al, "Evaluation of the LANL hand held multiplicity shift register and Canberra JSR-15," NSS/MIC IEEE Conference Proceedings, Honolulu, Hawaii, October 28-November 3, 2007 Paper \# 1894.

[16] B. Harker, M. Kirk, "INCC Software Users Manual," Los Alamos National Laboratory Report, LA-UR-01-6761, March 2009.

[17] H.O. Menlove, J. Phillips, et al. "Apparatus for in situ Determination of Burnup, Cooling Time and Fissile Content of an Irradiated Nuclear Fuel Assembly in a Fuel Storage Pond," U.S. Patent 4,510,117, Washington D.C., April 9, 1985. 